\title{
ADAPTING INTERDISCIPLINARY MEMS TEACHING AND TRAINING IN A "SMALL" FACULTY ENVIRONMENT
}

\author{
Shekhar Bhansali \\ BioMEMS and Microsystems Laboratory, Department of Electrical Engineering, University of South Florida \\ 4202, E.Fowler Avenue, Tampa, FL 33620, USA
}

\begin{abstract}
This paper highlights the unique curriculum modifications that enable MEMS education to keep pace with changing industry dynamics even in a "small" faculty environment. In a rapidly widening MEMS discipline, the methods outlined address an increasing need to impart the diverse technical fundamentals within a single course. Suitable education in the field requires an interdisciplinary syllabus and thus necessitates an innovative curriculum. This paper outlines two course modules currently being taught at the University of South Florida that are based upon these educational improvements. This paper intends to initiate a discussion on what should be an accepted definition of a MEMS course and what is the market expectation of the skill set that a student acquires as an outcome of the course.
\end{abstract}

\section{INTRODUCTION}

Rapid advancements in the field of microsystems coupled with its continued relevance to diverse sensor applications have been the seed for current revolution in MEMS education. Changing industrial needs have broadened the required knowledge base of students. There is thus an increasing need to diversify the technological background on which MEMS courses are built.

In order to address the issue, universities with critical mass of faculty active in the area of MEMS have established programs with 5-7 MEMS related courses. Many engineering schools have multiple course offerings covering different aspects of fundamentals, design, processing, materials and application aspects, while others schools use few faculty to offer a sequence of MEMS courses. While some schools focus on rendering sequential courses dealing exclusively on the design and fabrication aspect of a MEMS device separately, many combine to deliver multiple specialized courses such as Optical MEMS, BioMEMS, etc. These courses are often listed as electives with the introductory course forming the core. Institutions with small faculty environment are not able to cater to the demands of the rapidly widening MEMS discipline by conventional course designs. These institutions have to combine selected functional modules to offer relatively few sequence of courses. This constraint has resulted in a specialized course design that attempts to incorporate the diversity and details of the technological topics within a single course.

The content of the courses designed at USF also addresses the fact that the next generation of student community would be required to pursue research/work in a collaborative and interdisciplinary manner to achieve scientific breakthroughs and solve complex problems. Various funding agencies promote interdisciplinary programs around MEMS that require cross disciplinary graduate and undergraduate education. Consequently, the USF MEMS curriculum has undergone a major revision resulting in the revamping of the underlying objectives that include "the cultivation of desire to understand and assimilate nondisciplinary information to implement new solutions using MEMS".
As a part of continued discussion, the MEMS courses offered in the department of Electrical Engineering at the University of South Florida are presented as case studies. The architecture of these specially designed modules with course features and evaluations are illustrated. The inherent challenges involved in teaching a course comprising a wide spectrum of audience are also highlighted.

\section{MEMS COURSES AT USF}

\section{A. Chem. /Bio Sensors \& Microfabrication}

USF's academic program in the area of bio-MEMS has grown significantly over the past five years and is the cornerstone of NSF sponsored IGERT and Bridge to the Doctorate programs at USF. The programs, designed to work at the intersections of various disciplines, draw students from diverse backgrounds: Engineering (Electrical, Chemical, Mechanical, Computer Science, and BioMedical), Medicine, Arts and Sciences (Chemistry, Physics, and Biology) and Marine Science. The first interdisciplinary course - 'Chemical/Biological Sensors and Microfabrication' has been designed to engage the diverse audience. The course has been designed for graduate and senior undergraduate students. The content has been designed to go start with concepts of various sciences by describing their role in state of the art MEMS devices. Theoretical concepts in physics, chemistry, biology, materials science, electrical engineering and mathematics are related to their practical implementation in these devices. Recognizing the span of concepts covered, students are not expected to have an all-inclusive background in the relevant sciences. Thus the course does not have any prerequisite requirements. However, exposure to introductory courses in physical/analytical chemistry is generally advised.

Unlike conventional course designs, this introductory course reviews and concentrates on building a strong foundation on various sensing principles (physical, chem., and bio) with emphasis on suitable micromachining techniques required for realtime microsystem implementation.

To ensure a continued engagement of the diverse student body, MEMS processes and strategies are studied in relation to specific environments, applications and conditions. This helps students understand the various dynamics involved beyond the scientific principle of operation. For example, the human skin is introduced as a smart interface to seamlessly couple multifunctional micromachined systems for useful data extraction. In this case, issues that arise as the micro system and blood and/or other fluids interact are discussed leading to discussions on materials and bio-compatibility. Next compatibility in the context of marine environment is discussed, leading students to dwell on the issues of design changes to respond to the environment. 
EEL 6935/4936 Chemical Biological Sensors and Microfabrication

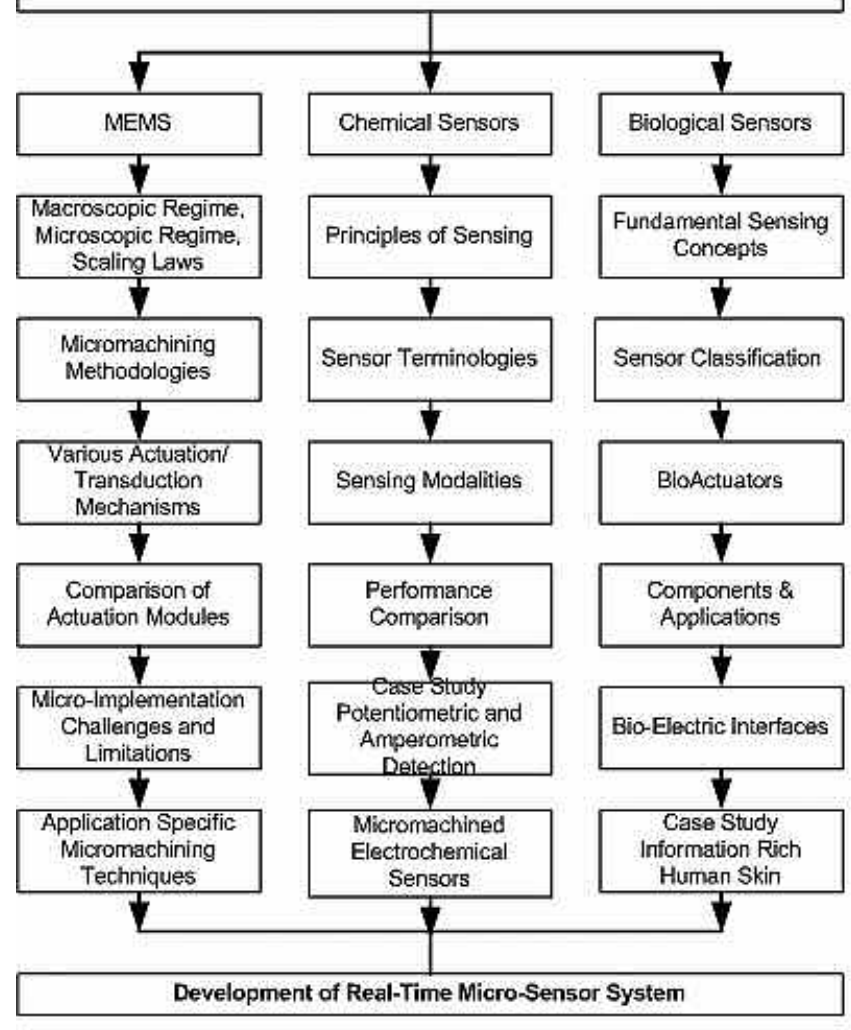

Figure 1. Flow Chart Illustrating the Design and Architecture of the Introductory Course

The course content is illustrated in Figure 1. It uses a topdown approach, i.e., understanding of how the system functions at the macroscopic level is gained first and then suitable approximations or modification to the existing theory are made at the microscopic level. Starting with the explanation of sensing principles across various domains, sensor classification and terminologies are described for students to understand industry relevant tolerance and specifications. Various sensing modalities are compared for students to appreciate the application specific suitability of each technique. Information on the different actuation/transduction mechanisms are provided, supplemented by their fabrication methods. In this course, the concepts are conveyed through the use of prototype models, handouts, visual aids, power point slides, guest lectures, paper reviews and in-house demo of processes.

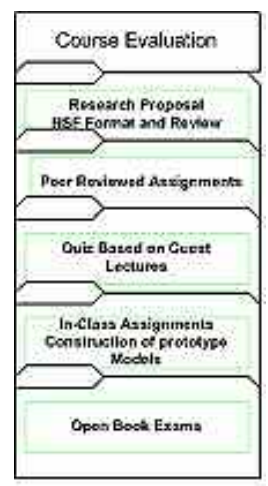

Figure 2: Course Evaluation Criteria for the Introductory Course
To leverage the diverse back ground of the class, students are asked to develop an NSF style proposal (figure 2) based on their idea of MEMS application that would be relevant in their field of expertise. The personal information is removed from the proposals and they undergo a panel review. The panel is formed of the students and moderated by the instructor. The students evaluate and rank the proposals with a clear understanding that only $15 \%$ of the proposals can be in the highly recommended category (i.e. get a $100 \%$ ) score. This unique andragogic exercise serves to enhance the thought process while enabling students to learn more about different areas of interest. Panel discussions facilitate constructive criticisms with appraisal of the subject while drafting proposals improves technical writing, a critical skill set required by the corporate world.

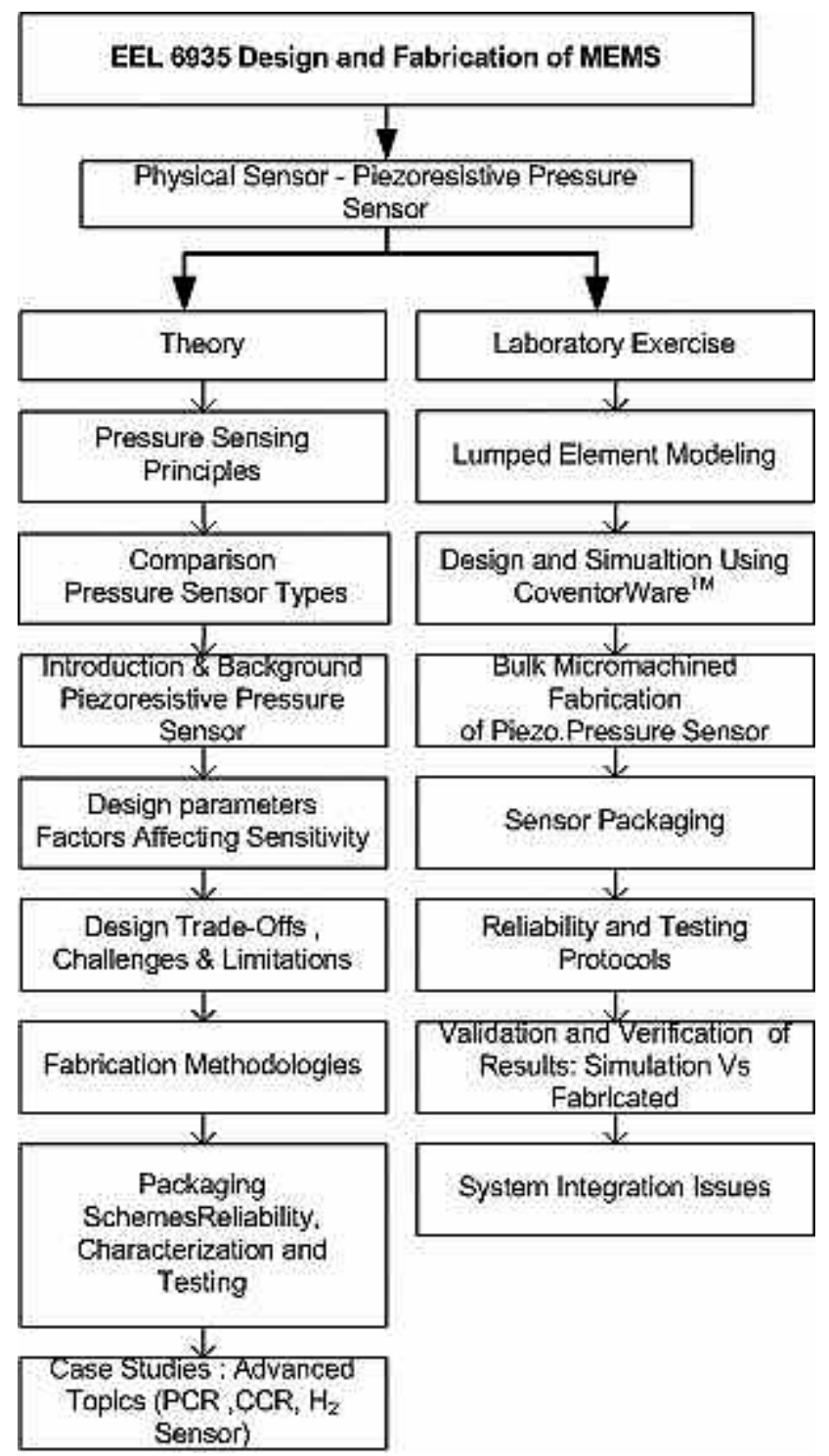

Figure 3: Flow Chart Illustrating the Architecture of the Advanced MEMS Course 


\section{OUTCOMES AND CHALLENGES}

Over the past three years the students have consistently stated that there is too much and too diverse reading involved. They have rated the panel discussion as the single biggest learning experience as it helps them understand the different perspectives of the same issues and also how people feel. One of the foremost challenges in teaching this course has been the absence of a single prescribed textbook. Students are provided with and advised to follow tailor made handouts. They are encouraged to study in interdisciplinary teams. This is facilitated by requiring them to review and document the current state of the art technology published in papers and patents. The course continues to be team taught and the integration of sciences and engineering continues to be challenge.

\section{B. Design and Fabrication of MEMS}

This second course in the two course sequence, discusses the design aspects, provides students with hands on fabrication and looks at uses case studies in development of micro systems. The students build and test a pressure sensor namely a piezoresistive pressure sensor. This course facilitates the understanding of experimental validation of simulated results and design trade-offs to optimize system performance. Figure 3 illustrates a flow-chart describing the course structure.

The course aims at developing a skill set for applying the fundamental concepts of MEMS in (a) designing and modeling MEMS based piezoresistive pressure sensor, and (b) fabrication and testing of the pressure sensor. The design component of the course requires the use of CoventorWare ${ }^{\mathrm{TM}} 2005$ to create, simulate and refine device models. Implementation of a predesigned device in a clean room environment imparts hands-on experience in fabrication. Case studies include advanced topics from microfluidics such as micromachined PCR (Polymerase Chain Reaction) reactors, optical devices like CCRs (Corner Cube Retro Reflectors), geared mirrors and microhotplate $\mathrm{H}_{2}$ gas sensor systems. These special modules emphasize on the design and fabrication constraints from a system's perspective.

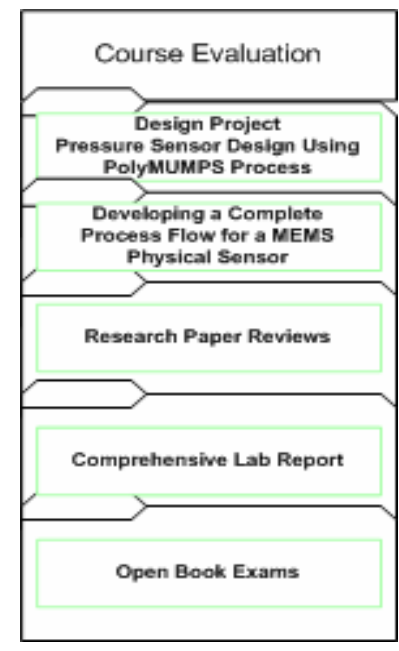

Figure 4: Course Evaluation Criteria for the Advanced Course
As part of evaluation criteria (figure 4), the students are asked to design, simulate and validate a similar MEMS device/system using industry standard PolyMUMPS process with appropriate calibration and testing protocols.

\section{Outcomes}

The course contents and evaluation criteria are designed to impart the students with required skill set needed for survival in the fast paced sensor industry. With the completion of this course students are able to: a) identify and understand interactions across domains; b) analyze failure modes; c) account for the discrepancy between simulated and experimental values.

Most non-engineers find the conventions and conversions (e.g. lumped element modeling) the hardest to follow and have consistently shown a performance that lags mechanical engineers. The mathematical base of students from different disciplines is very different and continues to be a challenge.

\section{Conclusions}

The paper describes the two course MEMS sequence and the content of the courses being taught at USF. The courses attempt to cover diverse areas in detail. The success resulting from the courses (panel review) and the challenges (student diversity, student background and the volume of information) are presented with a goal of initiating a discussion on what should be the required content of a MEMS course.

\section{Acknowledgements}

The author acknowledges the support of Praveen Kumar Sekhar and Puneet Khanna, TAs in the preparation of this paper. The educational activites were developed under NSF awards CAREER: ECS-0239262 and IGERT: DGE-0221681 